\title{
Heparanase Level in the Microcirculation as a Possible Modulator of the Metastatic Process
}

Neta Nevo, ${ }^{\dagger}$ Shourouk Ghanem, ${ }^{*}$ Yonatan Crispel, ${ }^{*}$ Mifleh Tatour,${ }^{*}$ Haim Cohen, ${ }^{*}$ Inna Kogan, ${ }^{*}$ Myriam Ben-Arush, ${ }^{\dagger}$ and Yona Nadir*

From the Thrombosis and Hemostasis Unit, ${ }^{*}$ and The Joan and Sanford Weill Pediatric Hematology Oncology and Bone Marrow Transplantation Division, ${ }^{\dagger}$ Rambam Health Care Campus, The Bruce Rappaport Faculty of Medicine, Technion, Haifa, Israel

Accepted for publication April 25, 2019.

Address correspondence to Yona Nadir, M.D., Ph.D., Thrombosis and Hemostasis Unit, Rambam Health Care Campus, HaAliya HaShniya Street 8, PO Box 9602, Haifa 31096, Israel. E-mail: y nadir@rambam.health.gov.il.

\begin{abstract}
Metastasis most commonly occurs in the liver, lung, bone, and brain, implying its preference for specific organs. We hypothesized that organ microcirculation coagulation environment predisposes to tumor cell retention. Coagulation factors were analyzed using immunostaining, enzyme-linked immunosorbent assay, and heparanase procoagulant activity assay. In normal mice, expression levels of heparanase, tissue factor (TF), TF pathway inhibitor (TFPI), and TFPI-2 were low in the microcirculation of the liver, lung, brain cortex, and bone, and high in the microcirculation of the subcutis, skeletal muscle, brain subcortex, and bone marrow. C57BL/6 mice injected s.c. with B16 (F10) melanoma cells demonstrated lower levels of heparanase, TF, TFPI, and TFPI-2 in metastasis blood vessels compared to those in the primary tumor. In these mice with metastasis, liver and lung microcirculation turned to express high levels of coagulation proteins. Additionally, although mice with heparanase overexpression developed a larger primary tumor, they did not demonstrate a tendency for metastasis, as opposed to controls $(P<0.0001)$. Human umbilical vein endothelial cells, incubated with the B16 melanoma cell medium for 2 hours, expressed decreased levels of heparanase, TF, TFPI, and TFPI-2, and the effect was reversed by a peptide-inhibiting heparanase/TF complex interaction $(P<0.001)$. In summary, metastasis has a predilection to organs with low levels of heparanase, TF, TFPI, and TFPI-2 in the microcirculation, which enables tumor cell retention. Heparanase has a role in regulating the microcirculation milieu. (Am J Pathol 2019, 189: 1654-1663; https://doi.org/10.1016/j.ajpath.2019.04.019)
\end{abstract}

In activated endothelial and most nonendothelial cells, tissue factor (TF), heparanase (serving as a cofactor to TF), and TF pathway inhibitors (TFPI) 1 and 2 are present on the cell surface. Heparanase, a $\beta$-D-endoglucuronidase abundant in platelets and discovered 30 years ago, is an enzyme that cleaves heparan sulfate side chains on the cell surface and in the extracellular matrix. ${ }^{1,2}$ It is further recognized as a proinflammatory, proangiogenic, and prometastatic protein. . $^{3,4}$

Heparanase activity has been reported to correlate with the metastatic potential of tumor cells, which could be attributed to enhanced cell dissemination as a consequence of enzymatic heparan sulfate cleavage and remodeling of the extracellular matrix barrier. ${ }^{5}$ Previous clinical data have suggested a correlation between heparanase expression and metastasis. $^{5-8}$ Heparanase, lacking enzymatic activity due to point mutations (Glu225, Glu343) in its active site, has also been noted to exert nonenzymatic activity, independent of its involvement in extracellular matrix degradation. Enzymatically inactive heparanase has been shown to facilitate adhesion and migration of primary endothelial cells ${ }^{9}$ and to promote phosphorylation of signaling molecules such as Akt and SRC,, 10 the latter found to be involved in vascular endothelial growth factor A induction after exogenous addition of heparanase or its overexpression. ${ }^{9,11}$

Activation of the coagulation system in cancer patients is very common. Activated coagulation proteins not only contribute to clinical thrombotic manifestations but also serve as growth factors to tumor and endothelial cells. In addition, proteins released by the tumor, such as cytokines, can activate the coagulation system, imposing positive

Supported by Israel Science Foundation (ISF) Individual Research grant 656/15 (2016-2019).

Disclosures: None declared. 
feedback between cancer and coagulation. ${ }^{12}$ Evidence suggests that heparanase might also affect the hemostatic system in a nonenzymatic manner. ${ }^{13-15}$ Heparanase upregulates the expression of the blood coagulation initiator $\mathrm{TF}^{13}$ and interacts with TFPI on the cell surface membrane of endothelial and tumor cells, leading to dissociation of

A

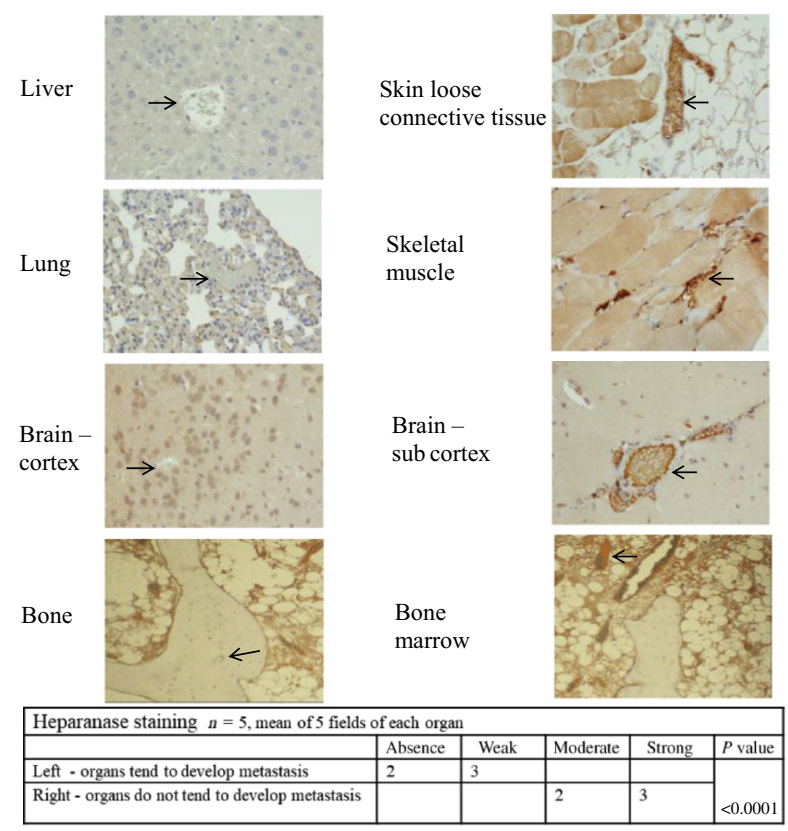

C

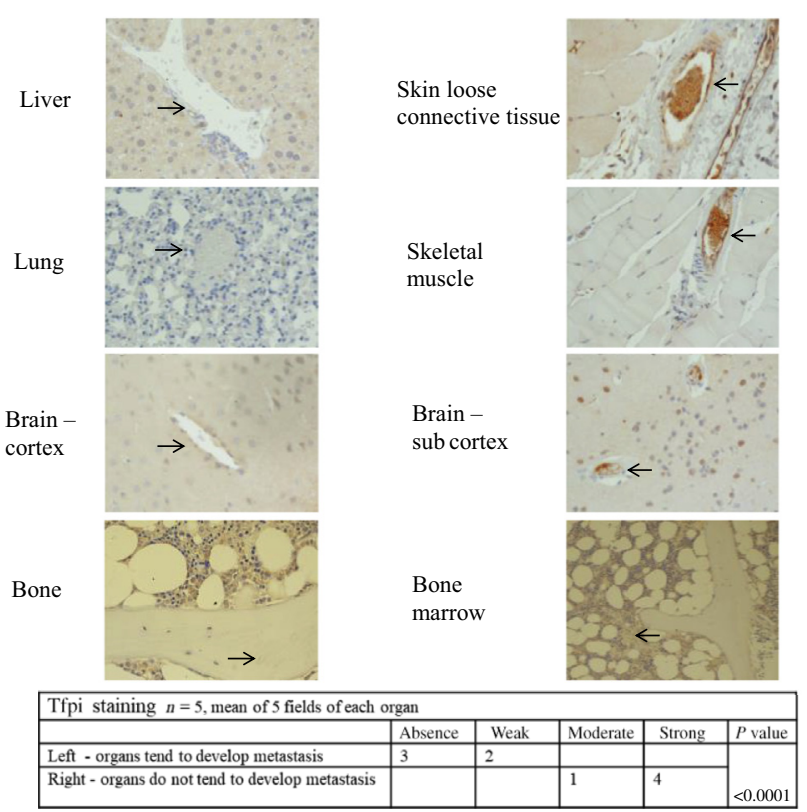

TFPI and resulting in increased cell surface-coagulation activity. ${ }^{14}$ Moreover, heparanase directly enhances TF activity, which leads to increased factor Xa production and subsequent activation of the coagulation system. ${ }^{15} \mathrm{We}$ recently described new peptides derived from the TFPI-2 first Kunitz domain, which inhibit the heparanase

B

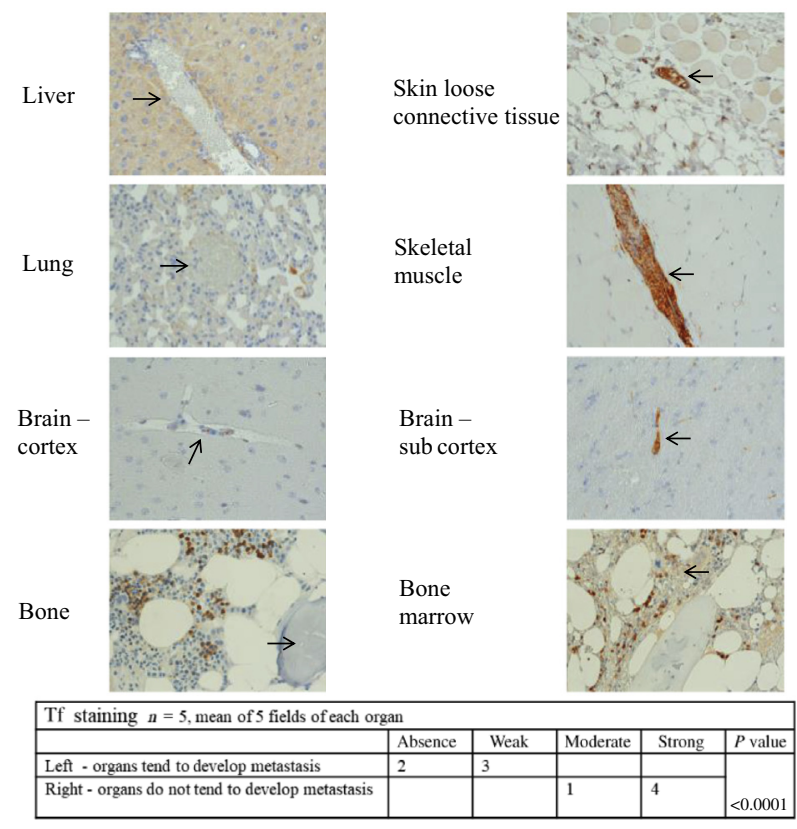

D

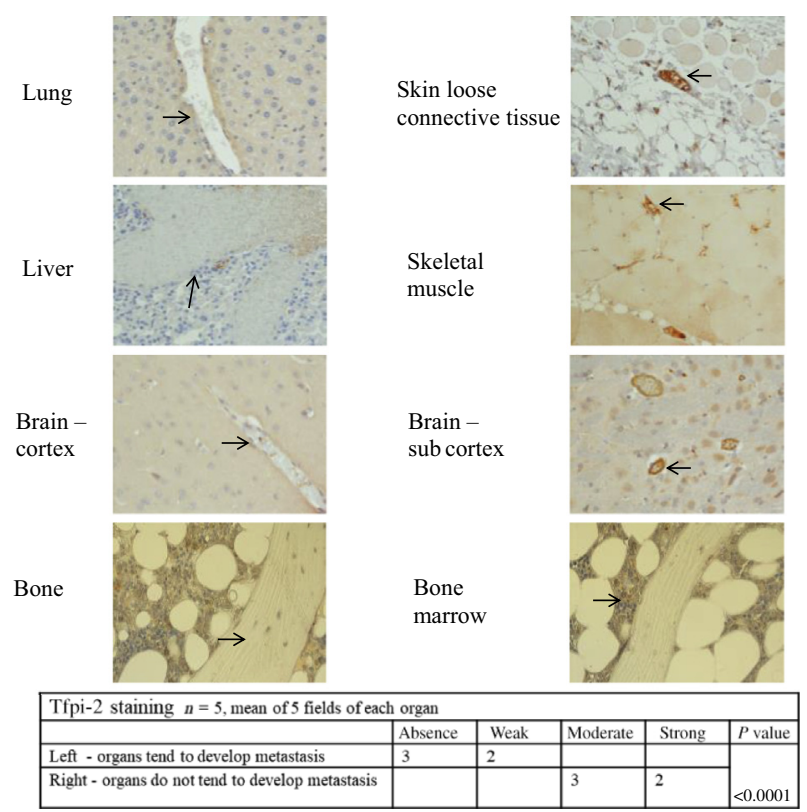

Figure 1 Levels of the coagulation proteins heparanase, tissue factor (TF), TF pathway inhibitor (TFPI), and TFPI-2 are decreased in the microcirculation of organs that tend to develop metastasis. Organs of five normal (without any genetic manipulation) ICR mice were immunostained. Levels of heparanase (A), TF (B), TFPI (C), and TFPI-2 (D) are decreased in the small blood vessels (arrows) of the liver, lung, brain cortex, and bone compared to the levels in the skin loose connective tissue, skeletal muscle, brain subcortex, and bone marrow. Representative images, captured with a Nikon E995 digital camera (Nikon, Tokyo, Japan). Tables below show staining intensity in organs that tend to develop metastasis versus organs that do not tend to develop metastasis. Significance was determined by $U$-test. Original magnification, $\times 50$ (A-D). 


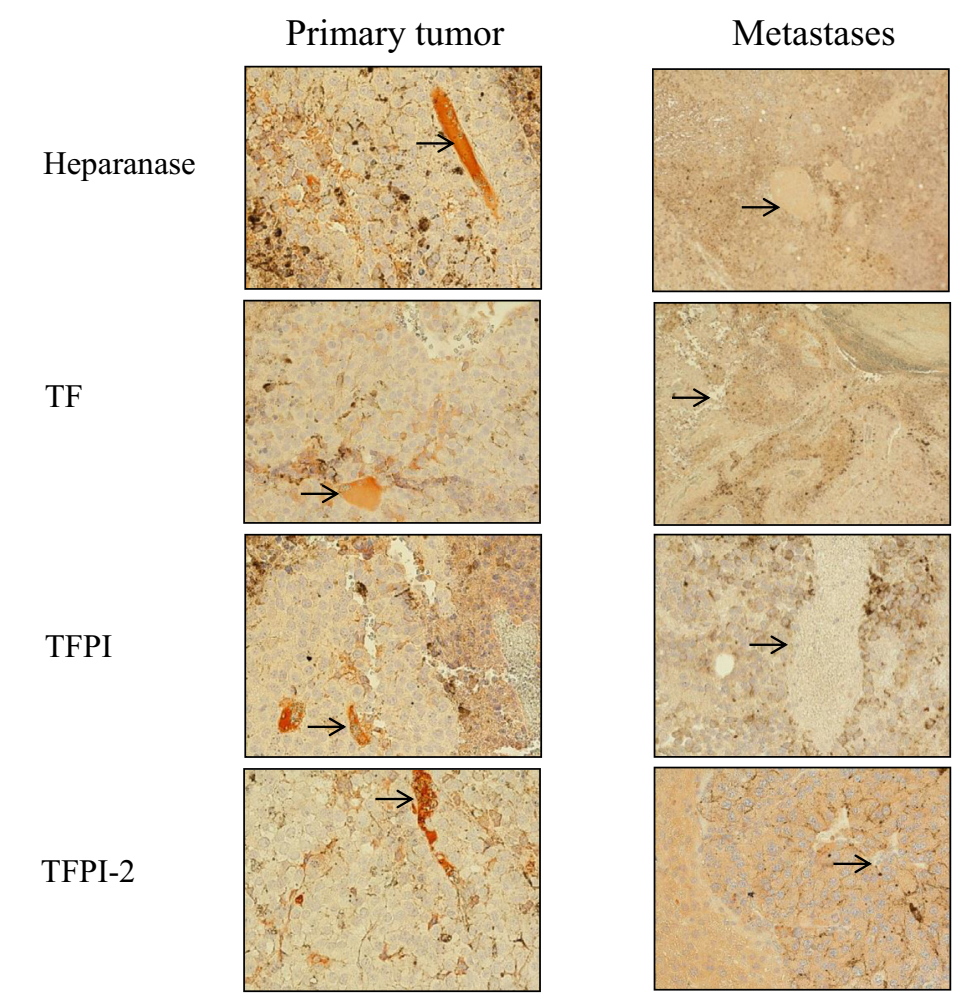

\begin{tabular}{|c|c|c|c|c|c|}
\hline \multicolumn{6}{|c|}{$n=5$, mean of 5 fields of each staining } \\
\hline & Absence & Weak & Moderate & Strong & $P$ value \\
\hline Left - primary tumor & & 1 & 2 & 2 & \\
\hline Right - metastases & 3 & 2 & & & $<0.001$ \\
\hline
\end{tabular}

Figure 2 Levels of coagulation factor are decreased in the blood vessels of metastasis compared to the levels in the blood vessels of primary tumor. An amount of $1 \times 10^{5}$ B16 (F10) melanoma cells were injected s.c. in the flank of five $\mathrm{C} 57 \mathrm{BL} / 6$ mice. Ten days later, the mice were sacrificed, and the primary tumor and metastases (four mice developed one or two metastases in the liver; one mouse, in the liver and lung) were compared. On immunostaining, the levels of the coagulation proteins heparanase, tissue factor (TF), Tf pathway inhibitor (TFPI), and TFPI-2 were lower in the blood vessels (arrows) of the metastases compared to the primary tumors. Metastases were evaluated in five different fields and were identified by cell morphology and black staining of melanin in the cells. Representative images, captured with a Nikon E995 digital camera (Nikon, Tokyo, Japan). Table below shows staining intensity in primary tumor versus metastases. Significance was determined by U-test. Original magnification, $\times 50$. procoagulant activity by obstructing the heparanase-TF complex. In vivo, the newly identified peptides attenuate activation of the coagulation system and reduce sepsis severity, without predisposing to significant bleeding tendency. ${ }^{16}$ In addition, the peptides inhibiting heparanase procoagulant activity significantly reduce tumor growth, vascularization, and relapse. ${ }^{17}$

Since the majority of cancer-related deaths are associated with metastases, the most dramatic improvements in morbidity and mortality result from the elimination of such disseminated disease. Metastases tend to have a predilection to specific organs. The liver, lungs, brain, and bones display the highest prevalences of metastatic growth. Other organs, such as muscles and skin, do not tend to develop metastases. ${ }^{18,19}$ We hypothesized that the endothelial cell surface-coagulation milieu of an organ predisposes to cell retention and the development of metastasis, and that heparanase may have a role in regulating the cell surface milieu.

\section{Materials and Methods}

\section{Mouse Model}

The study protocol was approved by the Technion Ethics Committee for Animal Research, and the procedures were conducted in accordance with institutional guidelines. Five normal ICR mice were scarified and their organs were evaluated. C57BL/6 mice were injected s.c. in the flank area with the highly metastatic melanoma cell line B16 (F10). A heparanase transgenic mouse model (background C57BL/6 mouse), in which human heparanase expression is driven by the $\beta$-actin promotor, thus enabling high levels of expression in essentially all tissues, ${ }^{20}$ was utilized. All of the experiments were performed in 7- to 8-week-old male mice to avoid age and hormonal effects.

\section{Reagents and Antibodies}

A single-chain GS3 heparanase gene construct, comprising the 8 - and $50-\mathrm{kDa}$ heparanase subunits $(8+50)$, was purified from the conditioned medium of baculovirus-infected cells. GS3 heparanase was assayed for the presence of bacterial endotoxin (Biological Industries, Beit Haemek, Israel) using the gel-clot technique (limulus amoebocyte lysate test) and was found to contain $<10 \mathrm{pg} / \mathrm{mL}$ of the endotoxin. ${ }^{13}$ Polyclonal antibody 1453 was raised in rabbits against the entire $65-\mathrm{kDa}$ heparanase precursor isolated from the conditioned medium of heparanase-transfected HEK-293 cells. The antibody was affinity-purified on immobilized bacterially expressed $50-\mathrm{kDa}$ heparanase glutathione-S-transferase 


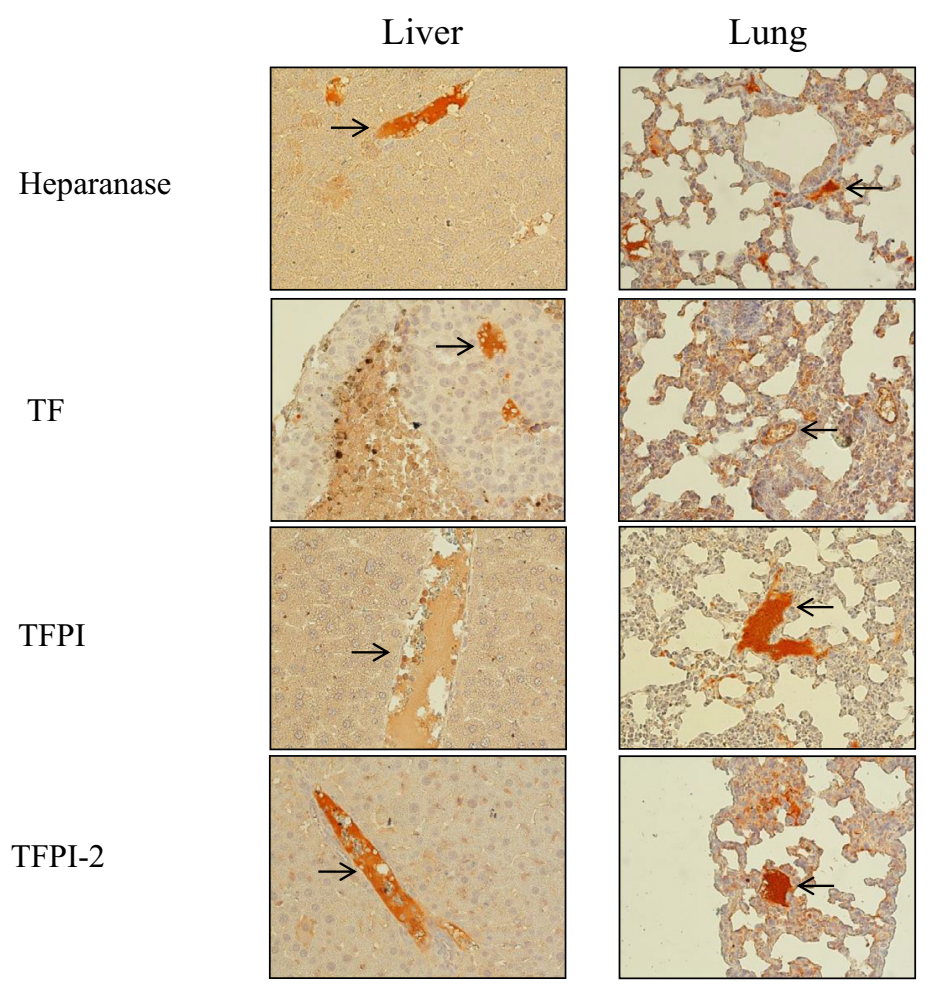

$n=5$, mean of 5 fields of each staining

\begin{tabular}{|l|l|l|l|l|l|}
\hline & Absence & Weak & Moderate & Strong & \\
\hline Left - liver & & & 2 & 3 \\
\hline Right - lung & & & 2 & 3 \\
\hline
\end{tabular}

Figure 3 Blood vessels in the organs tending to develop metastasis exhibit high levels of the coagulation proteins heparanase, tissue factor (TF), TF pathway inhibitor (TFPI), and TFPI-2 in mice with metastases. Microcirculation of the liver and lungs of the mice from the experiment in Figure 2 displays high levels of heparanase, TF, TFPI, and TFPI-2 (arrows), once the mouse has an advanced tumor with multiple metastases. Normal levels of the coagulation proteins in the microcirculation of the liver and lungs are shown in Figure 1. Representative images captured with a Nikon E995 digital camera (Nikon, Tokyo, Japan). Table below shows staining intensity in lung and liver of mice with metastases. $P=0.0001$ versus liver and lung staining in normal mice (Figure 1) (U-test). Original magnification, $\times 50$. fusion protein. ${ }^{21}$ Monoclonal antiheparanase antibody 1E1 was generated by immunizing BALB/C mice with the entire $65-\mathrm{kDa}$ heparanase protein. Antibody 733 was raised in rabbits against a $15-$ amino acid peptide that maps at the $\mathrm{N}$-terminus of the $50-\mathrm{kDa}$ heparanase subunit. ${ }^{21}$ Heparanase antibodies were kindly provided by Prof. Israel Vlodavsky and Dr. Neta Ilan (The Technion, Haifa, Israel). Recombinant human factor VIIa and plasma-derived human factor X were purchased from Sekisui Diagnostics, Inc. (Stamford, CT). All coagulation factors were dissolved in double-distilled water. Chromogenic substrate to factor Xa (I.D. 222; solubility, Tris buffer; $\mathrm{pH}$-8.4) was purchased from Sekisui Diagnostics. Bovine factor Xa was obtained from Sigma-Aldrich Corp (St. Louis, MO). Polyclonal anti-human TF and polyclonal antihuman TFPI antibodies were purchased from Santa Cruz Biotechnology (Santa Cruz, CA). Polyclonal anti-TFPI-2 was purchased from Bioss Antibodies (Woburn, MA).

\section{Cell Culture}

B16 (F10) mouse melanoma, EMT6 mouse breast carcinoma, MDA-231 human breast carcinoma, T47D human breast carcinoma, U87 human glioma, and human umbilical vein endothelial cells (HUVECs) were purchased from the
ATCC (Manassas, VA). Tumor cells were grown in the Dulbecco's modified Eagle's medium (Biological Industries, Beit Haemek, Israel) supplemented with $10 \%$ fetal calf serum and antibiotics. HUVECs were grown in the M199 medium (Biological Industries) supplemented with 20\% fetal calf serum and antibiotics.

\section{Histology Stains}

Staining of formalin-fixed, paraffin-embedded 5-micron sections was performed. Slides were deparaffinized with xylene and rehydrated, and endogenous peroxidase activity was quenched for 30 minutes by $3 \%$ hydrogen peroxide in methanol. Slides were then subjected to antigen retrieval by boiling (20 minutes) in $10 \mathrm{mmol} / \mathrm{L}$ citrate buffer, $\mathrm{pH} 6$. Slides were incubated with $10 \%$ normal goat serum in phosphate-buffered saline for 60 minutes to block nonspecific binding, followed by incubation $\left(20\right.$ hours, $\left.4^{\circ} \mathrm{C}\right)$ with anti-TF, anti-TFPI, anti-TFPI-2 or anti-heparanase 733 antibodies, diluted 1:100 in blocking solution. Slides were then extensively washed with phosphate-buffered saline containing $0.01 \%$ Triton X-100 and incubated with a secondary reagent (EnVision kit; Dako, Glostrup, Denmark) or secondary fluorescent reagent (Jackson ImmunoResearch 

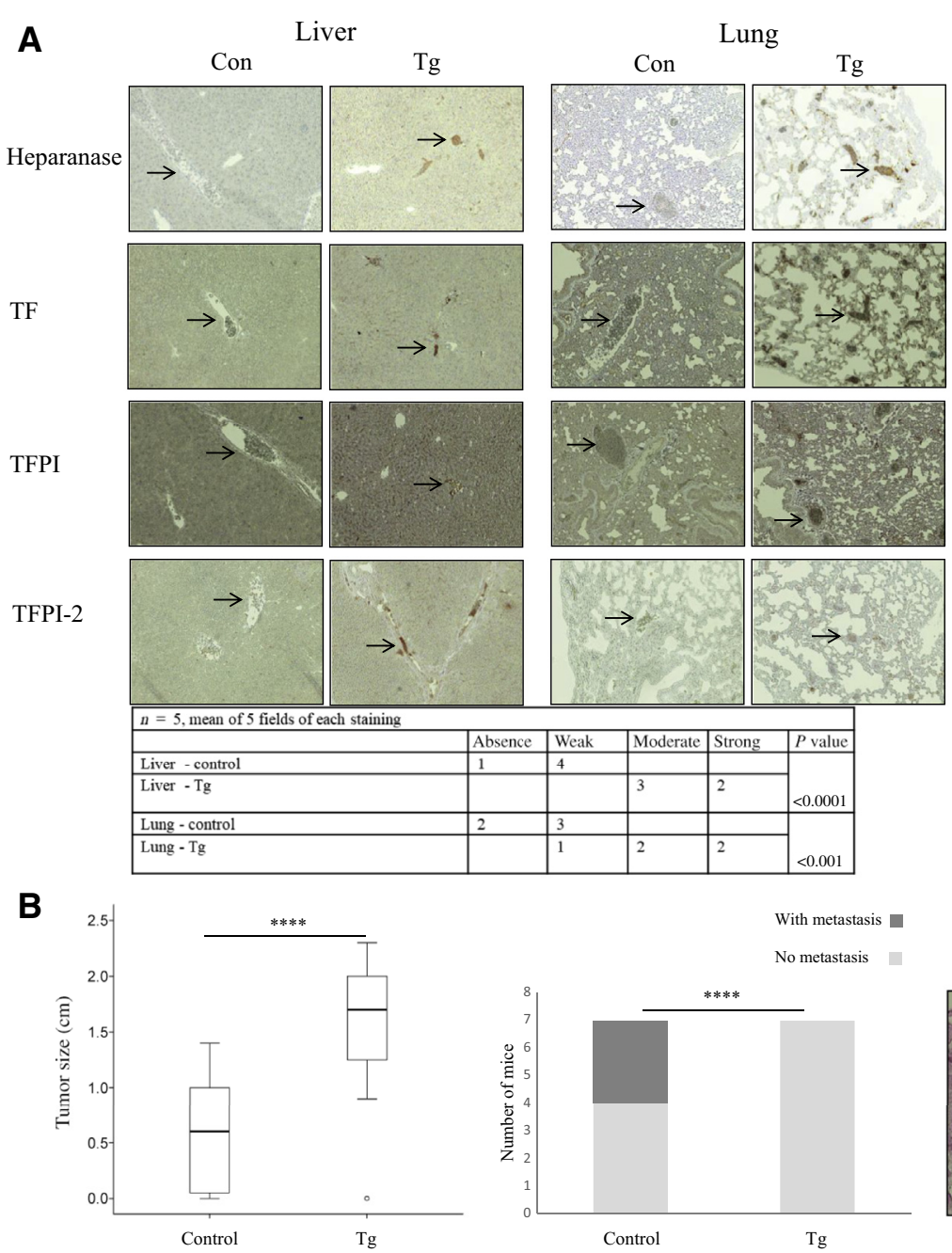

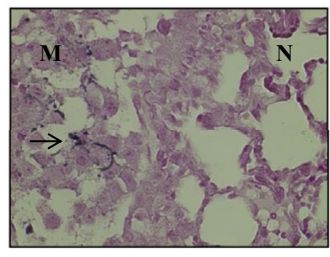

Lung

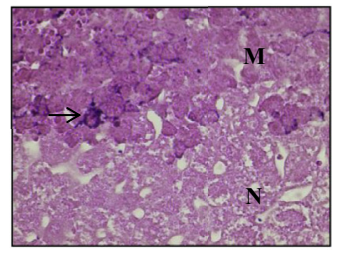

Liver

Figure 4 High levels of the coagulation proteins heparanase, tissue factor (TF), Tf pathway inhibitor (TFPI), and TFPI-2 in the microcirculation protect against metastasis. A: Levels of the heparanase, TF, TFPI, and TFPI-2 are higher in the liver and lung microcirculation of heparanase-treated transgenic mice ( $\mathrm{Tg})$ that overexpress heparanase (C57BL/6 background) compared to those in control C57BL/6 mice (Con) (arrows). Representative images. Table below shows staining intensity in liver and lung of mice. B: To assess the effect of the coagulation milieu on the ability to form metastasis, B16 (F10) melanoma cells $\left(1 \times 10^{5}\right.$ cells per mouse) were injected s.c. to the flank of seven transgenic mice and seven control animals. After 10 days, the mice were sacrificed. In the transgenic mice, the primary tumor is significantly larger compared to that in controls (box plots show the changes in parameters). No metastases are detected in the liver and lung. In the control group, three of seven mice developed one or two metastases (two mice in the liver and lung; one mouse, in the liver only). Metastases were evaluated in 10 different fields. Representative images show lung and liver metastases stained specifically for melanin by Fontana-Masson staining (arrows) in control mice (Nikon E995 digital camera; Nikon, Tokyo, Japan). Data are expressed as medians (25th to 75th percentiles, 10th to 90th percentiles). ${ }^{* * * *} P<0.0001$ (U-test). Original magnification, $\times 50(\mathbf{A}$ and $\mathbf{B}) . M$, metastasis; $\mathrm{N}$, normal tissue.

Laboratories, West Grove, PA) according to the manufacturer's instructions. After additional washes, color was developed with the AEC reagent (Sigma-Aldrich). FontanaMasson staining was performed according to the manufacturer's instructions (Sigma-Aldrich). Tissue immunohistochemistry results were analyzed by two of the authors (N.N., S.G.) unaware of the slide allocation. Discrepancies in the analyses were reconciled after assessment by a third reviewer (Y.C.). Five high-power fields were evaluated in each stained slide. Staining intensity was scored as follows: 0 indicates no staining; 1 indicates weak intensity; 2 indicates moderate intensity; and 3 indicates marked intensity. Representative intensity of staining was shown.

\section{Heparanase ELISA}

Wells of microtiter plates were coated $\left(18\right.$ hours, $\left.48^{\circ} \mathrm{C}\right)$ with $2 \mu \mathrm{g} / \mathrm{mL}$ of anti-heparanase monoclonal antibody $1 \mathrm{E} 1$ in 50 $\mu \mathrm{L}$ of coating buffer $\left(0.05 \mathrm{~mol} / \mathrm{L} \mathrm{Na} \mathrm{Na}_{3}, 0.05 \mathrm{~mol} / \mathrm{L}\right.$ $\mathrm{NaHCO}_{3}, \mathrm{pH}$ 9.6) and were then blocked with $2 \%$ bovine serum albumin in phosphate-buffered saline for 1 hour at $37^{\circ} \mathrm{C}$. Samples $(200 \mu \mathrm{L})$ were loaded in triplicate and incubated for 2 hours at room temperature, followed by the addition of $100 \mu \mathrm{L}$ of antibody $1453(1 \mu \mathrm{L} / \mathrm{mL})$ for an additional period of 2 hours at room temperature. horseradish peroxidase-conjugated goat anti-rabbit $\mathrm{IgG}$ $(1: 20,000)$ in blocking buffer was added (1 hour, room 
Microcirculation of primary tumor

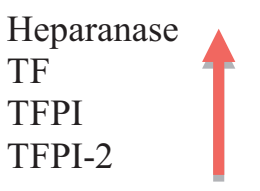

Microcirculation of organs enabling metastasis

Heparanase
TF
TFPI
TFPI-2

Shift in microcirculation of organs enabling metastasis

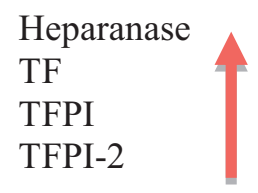

Microcirculation in metastasis

Heparanase
TF
TFPI
TFPI-2

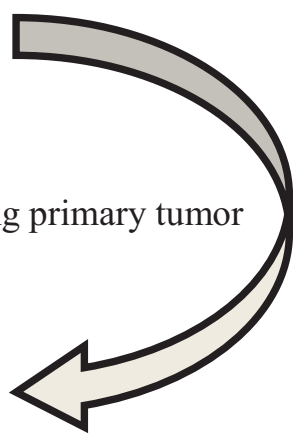

Figure 5 A model of metastasis organ predilection. The primary tumor demonstrates a high level of the assessed coagulation parameters, as demonstrated in Figure 2, whereas the metastases and the organs promoting metastases have low levels of these coagulation proteins, as shown in Figures 1 and 2. According to Figure 3, after tumor growth, there is a shift in the coagulation protein presentation, potentially hampering the occurrence of new metastases but enabling further local growth similar to that observed in the primary tumor.

temperature), and the reaction was visualized by the addition of $50 \mu \mathrm{L}$ of chromogenic substrate (TMB; MP Biomedicals, Eschwege, Germany) for 30 minutes. The reaction was stopped with $100 \mu \mathrm{L}$ of $\mathrm{H}_{2} \mathrm{SO}_{4}$, and absorbance at 450 $\mathrm{nm}$ was measured using an enzyme-linked immunosorbent assay (ELISA) plate reader (PowerWave XS; BioTek Instruments, Winooski, VT). Plates were washed five times with a washing buffer [phosphate-buffered saline, $\mathrm{pH}$ 7.4, containing $0.1 \%$ (w/v) Tween 20] after each step. As a reference for quantification, a standard curve was established by serial dilutions of recombinant $8+50$ GS3 heparanase $(390 \mathrm{pg} / \mathrm{mL}$ to $25 \mathrm{ng} / \mathrm{mL}){ }^{22}$

\section{Statistical Analysis}

Data were evaluated using SPSS software version 13.0 (SPSS Inc., Chicago, IL). Statistics was calculated by the nonparametric $U$-test. Values are reported as medians and ranges. The significance level was set at $P<0.01$.

\section{Results}

Levels of the Coagulation Proteins Heparanase, TF, TFPI, and TFPI-2 in the Microcirculation of Organs that Tend to Develop Metastasis

Organs of five ICR mice (normal mice, without genetic manipulation) were immunostained. Levels of the coagulation parameters heparanase, TF, TFPI, and TFPI-2 were significantly decreased in the microcirculation of the liver, lung, brain cortex, and bone compared to the skin loose connective tissue, skeletal muscle, brain subcortex, and bone marrow
(Figure 1). Other organs that did not tend to harbor metastasis, including kidney (medulla microcirculation staining was more prominent than that in the cortex), heart, and intestine submucosa, were also analyzed and high levels of expression of heparanase, TF, TFPI, and TFPI-2 were found in the microcirculation of these organs (data not shown).

Levels of Heparanase, TF, TFPI, and TFPI-2 in Blood Vessels of Metastasis Compared with Primary Tumor

Highly metastatic B16 (F10) melanoma cells $\left(1 \times 10^{5}\right)$ were injected s.c. to the flank of $5 \mathrm{C} 57 \mathrm{BL} / 6$ mice. Ten days later, the mice were sacrificed and the primary tumor and metastasis were compared. Immunostaining to heparanase, TF, TFPI, and TFPI- 2 in the blood vessels revealed that these protein levels were higher in the blood vessels of the primary tumor compared to the metastasis. Metastases were evaluated in the liver and lung tissues (Figure 2).

Levels of Heparanase, TF, TFPI, and TFPI-2 in the Microcirculation of the Organs Tending to Develop Metastasis in Metastatic Mice

Microcirculation in the liver and lungs demonstrated high levels of heparanase, TF, TFPI, and TFPI-2 once the mice had advanced tumor with multiple metastases (Figure 3).

Heparanase, TF, TFPI, and TFPI-2 in the Microcirculation Protect from Metastasis

Our assessment of the levels of heparanase, TF, TFPI, and TFPI-2 in various tissues, including liver, lung, brain, 
skin loose connective tissue, skeletal muscle, bone, and bone marrow, showed that, while mice with heparanase overexpression had lower levels of heparanase, TF, TFPI, and TFPI-2 in the organs tending to develop metastasis, the levels still appeared to be higher compared to those observed in control mice. Representative figures demonstrating the higher levels of heparanase, TF, TFPI, and TFPI-2 in the lung and liver microcirculation are presented in Figure 4A. To explore the impact of the coagulation milieu on the ability to form metastasis, B16 (F10) melanoma cells $\left(1 \times 10^{5}\right.$ cells per mouse $)$ were injected s.c. into the flank of seven heparanase transgenic mice and seven controls. After 10 days, the mice were sacrificed. In the heparanase-treated transgenic mice, the primary tumor was significantly larger compared to that in controls $(P<0.0001)$, but no metastases developed in the liver or lung $(P<0.0001$ and $P<0.001$, respectively), as shown in Figure 4B.

The results are summarized in Figure 5.

\section{Effects of B16 Melanoma Medium Mimicking Tumor Environment on Heparanase Levels in HUVECs}

In light of the results in the heparanase transgenic mice (Figure 4A), the regulatory effect of heparanase on the intracellular level and release of proteins TF, TFPI, and TFPI-2 was explored. The medium derived from 48-hour incubation with $80 \%$ confluence of B16 (F10) melanoma cells was added to endothelial cells for 2-hour incubation. The heparanase level, as estimated by ELISA, was decreased in these cells compared to that observed after incubation with full normal medium (Figure 6A). This result was further verified by immunostaining. In the same experiment, reduced levels of TF, TFPI, and TFPI-2 in HUVEC were found (Figure 6B).

\section{Effect of Inhibition of Heparanase on the Release of Heparanase, TF, TFPI, and TFPI-2 from HUVECs and Tumor Cells}

We suspected that heparanase in the tumor medium induced the release of the proteins. To verify the specific effect of heparanase, HUVECs were incubated for 2 hours with B16 (F10) melanoma cell medium, and a peptide, which we generated $^{16}$ and which inhibits the interaction between TF and heparanase (peptide 7), was added. The level of heparanase in HUVECs was significantly decreased compared to that in control $(P<0.001)$. The effect was reversed when peptide 7 was added to the medium (Figure 7A). Similar results were obtained when HUVECs were incubated in the medium of T47D cells (Figure 7B). When MDA-231 tumor cells were incubated with the B16 (F10) melanoma cell medium, peptide 7 inhibited the decrease in heparanase in the cells in a dose-dependent manner, as demonstrated by ELISA (Figure 7C). Similar results were shown on immunostaining of U87 cells. Remarkably, peptide 7 also
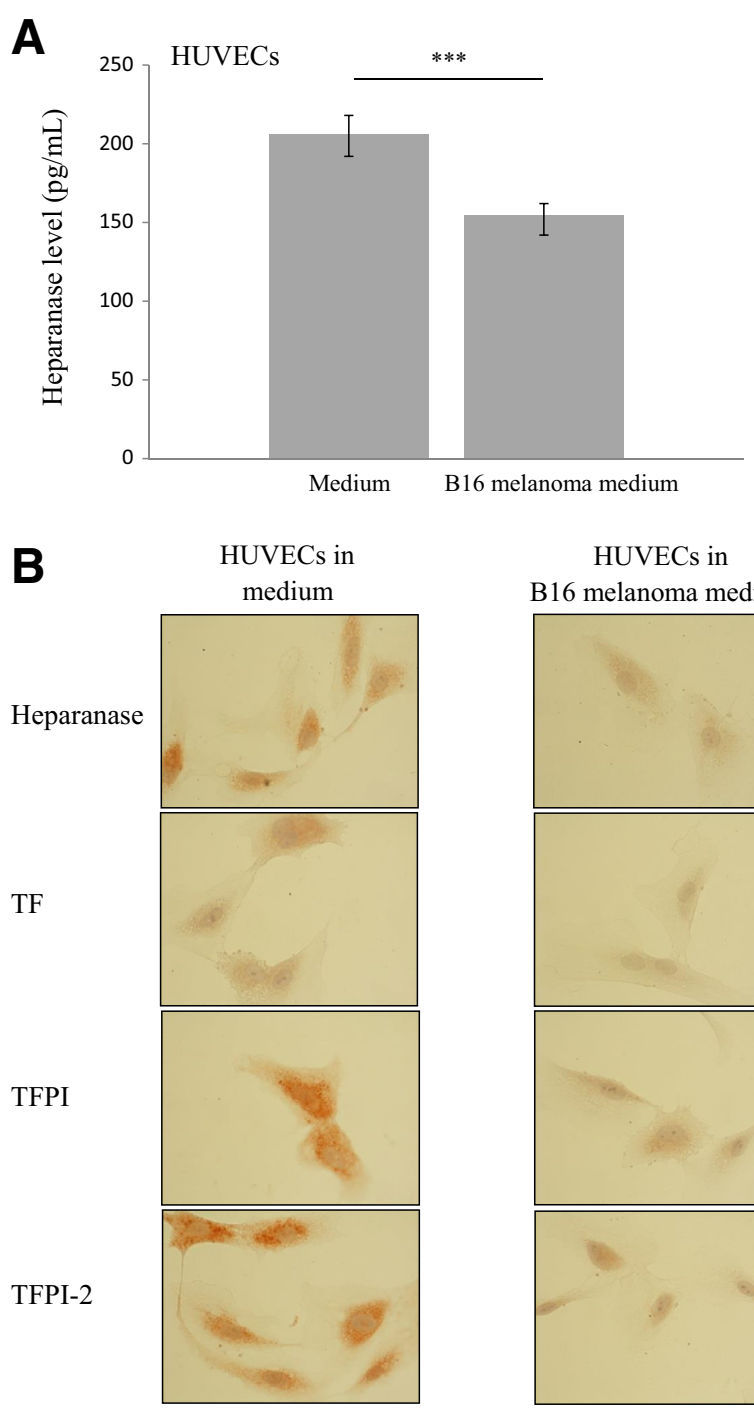

HUVECs in B16 melanoma medium

Figure 6 Effects of B16 (F10) melanoma medium mimicking tumor environment on heparanase level in human umbilical vein endothelial cells (HUVECs). Medium with $10 \%$ fetal calf serum derived from 48-hour incubation in $80 \%$ cell confluence of B16 (F10) mouse melanoma cells was added to endothelial cells for 2-hour incubation. A: Levels of heparanase, as determined by enzyme-linked immunosorbent assay, are decreased in these cells compared to those in cells incubated with normal full medium. B: Results are verified by evaluating levels of heparanase, tissue factor (TF), TF pathway inhibitor (TFPI), and TFPI-2 using immunostaining. Representative images, captured with a Nikon E995 digital camera (Nikon, Tokyo, Japan). These results suggest that the B16 (F10) medium contains molecules that induce the release of heparanase, TF, TFPI, and TFPI-2 from HUVECs. Data are expressed as medians (ranges) of triplicate measurements. ${ }^{* * *} P<0.001$. Original magnification, $\times 50$.

inhibited the decreases in TF, TFPI, and TFPI-2 levels in the cells (Figure 7D).

\section{Discussion}

The present study demonstrated a relationship between the organs tending to develop metastasis and low levels of certain coagulation factors, including heparanase, TF, TFPI, and TFPI-2, in the microcirculation. Moreover, such 
A

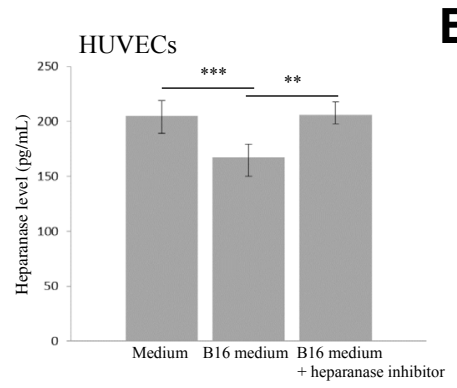

B

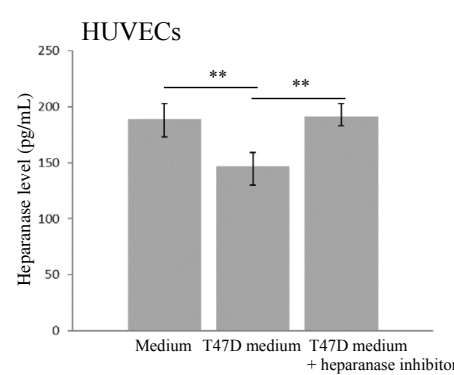

C

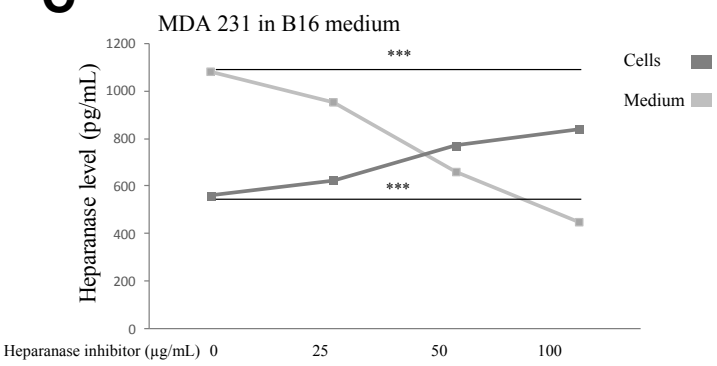

D

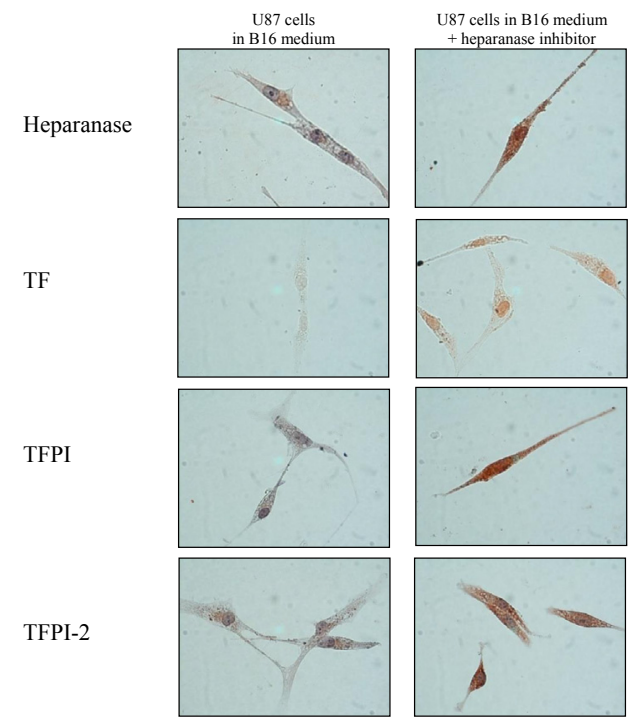

Figure 7 Inhibition of heparanase reverses the release of heparanase, tissue factor (TF), TF pathway inhibitor (TFPI), and TFPI-2 from human umbilical vein endothelial cells (HUVECs) and tumor cells. HUVECs were incubated with B16 (F10) melanoma cell medium and a peptide inhibiting the interaction between TF and heparanase (peptide 7, $50 \mu \mathrm{g} / \mathrm{mL}$ ) for 2 hours. A: Heparanase levels are significantly decreased in HUVEC lysates compared to controls. The effect is reversed when peptide 7 is added to the medium. B: Similar results are obtained when HUVECS are incubated in the medium of T47D human breast carcinoma cells. C: In tumor cells from MDA-231 human breast carcinoma incubated with B16 (F10) melanoma cell medium, peptide 7 inhibited the decrease in heparanase levels in a dose-dependent manner, as demonstrated by enzyme-linked immunosorbent assay. D: Similar results are shown in an additional tumor cell line, U87 human glioma, using immunostaining. Remarkably, peptide $7(50 \mu \mathrm{g} / \mathrm{mL})$ also inhibits the decreases in TF, TFPI, and TFPI-2 levels in the cells. These in vitro results support the role of heparanase in regulating the levels of the coagulation proteins TF, TFPI, TFPI-2 and heparanase in HUVECs and tumor cells. Representative images, captured with a Nikon E995 digital camera (Nikon, Tokyo, Japan). Data are expressed as medians (ranges) of triplicate measurements. ${ }^{* *} P<0.01$ and ${ }^{* *} P<0.001$. Original magnification, $\times 50$. reduction in the levels of these coagulation factors was observed in metastatic microcirculation only and not in the primary tumor. Remarkably, at the advanced tumor stage with multiple metastases, microcirculation in the aforementioned organs (Figure 1) was altered to that inhibiting further metastasis rather than enabling it (Figure 3). Results in mice with heparanase overexpression indicating a reduced ability to form metastasis in the liver and lungs strengthen our claim. Notably, in the mice with heparanase overexpression, the microcirculation levels of not only heparanase was increased but also TF, TFPI, and TFPI-2. We previously demonstrated that heparanase up-regulated the expression of TF and TFPI. ${ }^{13,14}$ We have presented evidence that TFPI-2 is also upregulated by heparanase. In vitro investigation of potential mechanisms involved in the control of microcirculation coagulation protein levels revealed that tumor medium contained molecules that induce the release of heparanase, TF, TFPI, and TFPI-2 from endothelial cells. In addition, the inhibition of heparanase interaction with TF using peptide 7 in endothelial and tumor cells reversed the effect of the tumor medium. Such findings may suggest a major role of heparanase in the regulation of these cell surface proteins. It could be assumed that as a tumor grows, heparanase from the primary tumor is likely to modify the microcirculation of the organs that normally have low levels of the coagulation proteins.
The mechanisms by which heparanase affects the other studied coagulation proteins are partially understood. Heparanase has been found to directly interact with TF and to upregulate its expression in endothelial cells. ${ }^{13,15}$ The effect of heparanase on TFPI release from the cell surface of endothelial and tumor cells was shown in our previous study, ${ }^{14}$ and a direct interaction between heparanase and TFPI- 2 was also recently demonstrated. ${ }^{16}$

In the experiments presented in Figures 6 and 7, the medium of B16 (F10) melanoma cells was used as an in vitro model of tumor environment affecting endothelial and tumor cells after 2 hours of incubation and it was found that the melanoma medium induced the release of heparanase, TF, TFPI, and TFPI-2 from these cells to the culture medium (mimicking the microcirculation), and that this effect was reversed when heparanase was inhibited. These experiments support suggestion of an effect of local heparanase on the endothelial and tumor cell microcirculation milieu. These results were further strengthened by the data obtained in mice with heparanase overexpression (Figure 4), demonstrating that a high level of heparanase in the microcirculation protects from metastasis. Nakajima et $\mathrm{al}^{23}$ found that heparanase heparan sulfate-degradative activity of metastatic B16 melanoma sublines correlated with their lung-colonizing potential. These results indicate that the level of heparanase in not only the organ 
microcirculation, but also in the primary tumor cells, affects the metastatic potential, as demonstrated in the present study. The data by Nakajima et $\mathrm{al}^{23}$ further enhance the findings on the major role of heparanase in the metastatic process.

The concept that organs present a different coagulation milieu in the blood vessels has been previously discussed. ${ }^{24}$ Is it possible that blood flow in the microcirculation enables the presence of different coagulation proteins? The blood flow velocity in the capillaries is estimated at 0.5 to $1 \mathrm{~mm} /$ second, whereas in the liver sinuses it is slower by about half. ${ }^{25}$ This time could be ample for the release or internalization of the evaluated coagulation proteins from the endothelial cells.

It is well documented that in metastatic solid tumors, such as lung, breast, and colon cancers, as well as melanoma, significantly improved cure rates have been demonstrated with drug treatment applied after surgical removal of the grossly apparent primary tumor compared to those achieved with either surgery or drug treatment when used alone. ${ }^{26}$ According to our findings, diminishing the primary tumor by surgery or chemotherapy can result in reversing the microcirculation of the organs enabling metastasis again. Hence, only a reduction of the primary tumor without further anticancer therapy has the potential to enhance metastasis. This interpretation could explain the clinically known course of tumor spread after primary tumor resection if no further treatment is given.

In conclusion, the current study was engendered by our intriguing previous finding regarding the level of four related cell surface-bound coagulation factors: heparanase, TF, TFPI, and TFPI-2. Organs tending to develop metastasis expressed low levels of these coagulation factors in the microcirculation, whereas organs that did not tend to harbor metastasis displayed high levels of these factors. In an attempt to shed light on this interesting finding, we revealed an involvement of heparanase in its own expression as well as in that of the three other factors in endothelial and tumor cells. Obviously, other mechanisms involved in the microcirculation expression of the cell surface coagulation proteins should be further investigated. Thus, the present study was the first to demonstrate a predilection of metastasis to the organs with low microcirculation levels of heparanase, TF, TFPI, and TFPI-2 (ie, liver and lung). Metastasis blood vessels appeared to exhibit lower levels of heparanase, TF, TFPI, and TFPI-2 compared to those of the primary tumor. In addition, in mice with metastasis, liver and lung microcirculation turned to express high levels of coagulation proteins. Finally, heparanase was found to have a role in regulating the microcirculation milieu. As metastasis is the major cause of death in cancer patients, the effect of a high endogenous heparanase level in the plasma on metastatic potential, and therapeutic strategies targeting the microcirculation coagulation milieu, warrant further research.

\section{Acknowledgments}

We thank Prof. Israel Vlodavsky and Dr. Neta Ilan (The Technion, Haifa, Israel) for providing the heparanase antibodies.

N.N. performed the research and analyzed the data; S.G., Y.C., M.T., H.C., and I.K. performed the research; M.B.-A. analyzed the data; Y.N. designed and performed the research, contributed vital new reagents or analytical tools, analyzed the data, and wrote the manuscript.

\section{References}

1. Freeman C, Parish CR: Human platelet heparanase: purification, characterization and catalytic activity. Biochem J 1998, 330: $1341-1350$

2. Pikas DS, Li JP, Vlodavsky I, Lindahl U: Substrate specificity of heparanases from human hepatoma and platelets. J Biol Chem 1998, 273:18770-18777

3. Parish CR, Freeman C, Hulett MD: Heparanase: a key enzyme involved in cell invasion. Biochim Biophys Acta 2001, 1471: M99-M108

4. Vlodavsky I, Friedmann Y: Molecular properties and involvement of heparanase in cancer metastasis and angiogenesis. J Clin Invest 2001, 108:341-347

5. Xu X, Quiros RM, Maxhimer JB, Jiang P, Marcinek R, Ain KB, Platt JL, Shen J, Gattuso P, Prinz RA: Inverse correlation between heparan sulfate composition and heparanase-1 gene expression in thyroid papillary carcinomas: a potential role in tumor metastasis. Clin Cancer Res 2003, 9:5968-5979

6. Maxhimer JB, Quiros RM, Stewart R, Dowlatshahi K, Gattuso P, Fan M, Prinz RA, Xu X: Heparanase-1 expression is associated with the metastatic potential of breast cancer. Surgery 2002, 132: 326-333

7. Liu LP, Sheng XP, Shuai TK, Zhao YX, Li B, Li YM: Helicobacter pylori promotes invasion and metastasis of gastric cancer by enhancing heparanase expression. World J Gastroenterol 2018, 24:4565-4577

8. Takaoka M, Naomoto Y, Ohkawa T, Uetsuka H, Shirakawa Y, Uno F, Fujiwara T, Gunduz M, Nagatsuka H, Nakajima M, Tanaka N, Haisa M: Heparanase expression correlates with invasion and poor prognosis in gastric cancers. Lab Invest 2003, 83:613-622

9. Gingis-Velitski S, Zetser A, Flugelman MY, Vlodavsky I, Ilan N: Heparanase induces endothelial cell migration via protein kinase B/Akt activation. J Biol Chem 2004, 279:23536-23541

10. Doviner V, Maly B, Kaplan V, Gingis-Velitski S, Ilan N, Vlodavsky I, Sherman Y: Spatial and temporal heparanase expression in colon mucosa throughout the adenoma-carcinoma sequence. Mod Pathol 2006, 19:878-888

11. Zetser A, Bashenko Y, Miao HQ, Vlodavsky I, Ilan N: Heparanase affects adhesive and tumorigenic potential of human glioma cells Cancer Res 2003, 63:7733-7741

12. Nadir Y, Brenner B: Cancer and thrombosis-new insights. Rambam Maimonides Med J 2018, 9. e0033

13. Nadir Y, Brenner B, Zetser A, Ilan N, Shafat I, Zcharia E, Goldshmidt O, Vlodavsky I: Heparanase induces tissue factor expression in vascular endothelial and cancer cells. J Thromb Haemost 2006, 4:2443-2451

14. Nadir Y, Brenner B, Gingis-Velitski S, Levy-Adam F, Ilan N, Zcharia E, Nadir E, Vlodavsky I: Heparanase induces tissue factor pathway inhibitor expression and extracellular accumulation in endothelial and tumor cells. Thromb Haemost 2008, 99:133-141

15. Nadir Y, Brenner B, Fux L, Shafat I, Attias J, Vlodavsky I: Heparanase enhances the generation of activated factor $\mathrm{X}$ in the presence of tissue factor and activated factor VII. Haematologica 2010, 95:1927-1934 
16. Axelman E, Henig I, Crispel Y, Attias J, Li JP, Brenner B, Vlodavsky I, Nadir Y: Novel peptides that inhibit heparanase activation of the coagulation system. Thromb Haemost 2014, 112:466-477

17. Crispel Y, Axelman E, Tatour M, Kogan I, Nevo N, Brenner B, Nadir Y: Peptides inhibiting heparanase procoagulant activity significantly reduce tumour growth and vascularisation in a mouse model. Thromb Haemost 2016, 116:669-678

18. LaBan MM, Nagarajan R, Riutta JC: Paucity of muscle metastasis in otherwise widely disseminated cancer: a conundrum. Am J Phys Med Rehabil 2010, 89:931-935

19. Hussein MR: Skin metastasis: a pathologist's perspective. J Cutan Pathol 2010, 37:e1-e20

20. Zcharia E, Metzger S, Chajek-ShaulL T, Aingorn H, Elikn M, Friedmann Y, Weinstein T, Jin-Ping L, Lindahl U, Vlodavsky I: Transgenic expression of mammalian heparanase uncovers physiological functions of heparan sulfate in tissue morphogenesis, vascularization, and feeding behavior. FASEB J 2004, 18:252-263
21. Zetser A, Levy-Adam F, Kaplan V, Gingis-Velitski S, Bashenko Y, Schubert S, Flugelman MY, Vlodavsky I, Ilan N: Processing and activation of latent heparanase occurs in lysosomes. J Cell Sci 2004, $117: 2249-2258$

22. Shafat I, Zcharia E, Nisman B, Nadir Y, Nakhoul F, Vlodavsky I, Ilan N: An ELISA method for the detection and quantification of human heparanase. Biochem Biophys Res Commun 2006, 341: 958-963

23. Nakajima M, Irimura T, Di Ferrante D, Di Ferrante N, Nicolson GL: Heparan sulfate degradation: relation to tumor invasive and metastatic properties of mouse B16 melanoma sublines. Science 1983, 220:611-613

24. Aird WC: Phenotypic heterogeneity of the endothelium: I. Structure, function, and mechanisms. Circ Res 2007, 100:158-173

25. Braet F, Wisse E: Structural and functional aspects of liver sinusoidal endothelial cell fenestrae: a review. Comp Hepatol 2002, 1:1

26. Laboratory models and clinical cancer. Dedicated to Dr. Frank M. Schabel, Jr. Cancer Treat Rep 1986, 70:1-229 